\title{
Results of Vitamin D Prescription in High Schools
}

\author{
Mahsa Houshdar* \\ Hooshdar Medical Technology Company, Iran
}

Received: September 04, 2017; Published: September 18, 2017

*Corresponding author: Mahsa Houshdar, Hooshdar Medical Technology Company, Iran-Karaj-Taleghani Ave-No 455-Second Floor, Iran, Tel: 009+832264770/00989354928132; Email: mhooshdar@gmail.com

\section{Abstract}

Introduction: Online Medicare is a method in which parts of a medical process - whether its diagnostics, monitoring or the treatment itself will be done by using online services. This system has been operated in 4 boys high school, 5 girl high schools.

Method: At the first step the students registered for using the system. It was not mandatory and not free. They participated in estimating depression scale, anxiety scale and clinical interview by online medical care system. During this estimation, we could find the existence and severity of depression and anxiety in each one of the participants, also we could find the consequent needs of each one, such as supportive therapy in mild depression or anxiety, need to visited by psychologist in moderate cases, need to visited by psychiatrist in moderate-severe cases, need to visited by psychiatrist and psychologist in severe cases and need to perform medical lab examination tests The lab examination tests were performed on persons specified by the system. The lab examinations were included: serum level of vitamin D, serum level of vitamin B12, serum level of calcium, fasting blood sugar, HbA1c, thyroid function tests and CBC. All of the students were solely treated by vitamins or minerals therapy and/ or treatment of medical problem (such as hypothyroidism). This system has been operated in high schools during 4 years. (April 2011 till March 2016). From Oct 2014 till May 2016, the ministry of education of Albors state of Iran has been given $5000 I U$ vitamin D3/ Monthly to every high schools students. During this period online medical care system had been operated in 6 high school of above average area of Karaj. Thus we had enough information to compare the prevalence of vitamin D deficiency (serum vitamin D level $<10$ ) and insufficiency (serum vitamin D level $>10 \&<30$ ) from the beginning of operation of the project till March 2016.

Results: Totally, the serum vitamin D3 level of 180 students have been measured from Oct 2014 till April 2015 and the results compare with the serum vitamin D3 level of 203 students have been measured from Oct 2015 till March 2016. The mean of vitamin D deficiency in academic year 2014-2015 was \%34.06 and in academic year 2015-2016, it was \%14.70. The mean of vitamin D insufficiency in academic year 2014-2015 was \%50.26 and in academic year 2015-2016, it was \%60.53. There was no any case of vitamin D toxicity. This results show that the prescription of 5000IU vitamin D3 can significantly decrease the range of vitamin D3 deficiency in high schools, but we need more time for decreasing the range of vitamin the insufficiency.

Conclusion: During two year prescription of 5000 IU vitamin D3/ monthly in high school, we face on 2.5 time decreasing of vitamin D deficiency and a few increasing of vitamin D insufficiency and no case of vitamin D toxicity.

\section{Introduction}

Online Medicare is a method in which parts of a medical process - whether its diagnostics, monitoring or the treatment itself will be done by using online services. At the first step the students were registered for using the system. They participated in estimating depression scale, anxiety scale and clinical interview by online medical care system. Then the lab examination tests were performed on persons specified by the system. The lab examinations include: serum level of vitamin D3\&4, serum level of vitamin B125\&6, fasting blood sugar7\&8, HbA1c7\&8, thyroid function test $9 \& 10$ and $\mathrm{CBC}$. All of the students were solely treated by vitamins or minerals therapy and/ or treatment of medical problems (such as hypothyroidism).

With operating this system during five years, we can get enough information for research about other issue. Beside we can see the changes of medical data and psychiatric data over time. The changes in social or economical situation can changes the severity of depression or anxiety in students and the results of every health political project that performed for recovery of medical or psychiatrical situation, spontaneously can be measured by the system.

\section{Method}

One of heath political project that have been performed during 2014 till 2016 academic years is : vitamin D3 prescription in high schools. Vitamin D3 prescription in high school is free. It have been given to student by the health coach of the high schools, but using of the tabs is not mandatory and no one would be punished for refusing of eating the tabs. Besides, we have been faced on a lot of advertisement $\mathrm{s}$ about the morbidity of vitamin D3 deficiency and the problem of high prevalence of this diseases during these years. 


\section{The dosage for prescription}

The health coach of every high school must give a tab of vitamin D3 monthly to every student. This tab contain of 5000IU vitamin D3. No primary research about the severity of vitamin D deficiency had been perform before vitamin D prescription. Whether the student use the tabs or not, is not the problem of the health coach, but public advertisement about the range of vitamin D deficiency and its morbidity, was a critical role in encouraging the students and their parents to use the tabs.

Hooshdar medical technology company research the The rate of medical diseases in the participants, chosen by the system for lab exam in six high school in one of above average neighborhoods of Karaj in Alborz state of Iran. Thus, this company have in control the information about the prevalence of vitamin D deficiency in academic year 2014-2015 and academic year 2015-2016 and their differences. It can partially interpret the results of prescription of vitamin D3 during two years, in Albors state of Iran.

\section{Results}

These tables show the prevalence of medical diseases in volunteers in every high school during academic year 2014-2015 \& 2014-2016 (Figures 1 \& 2) (Tables 1-7).

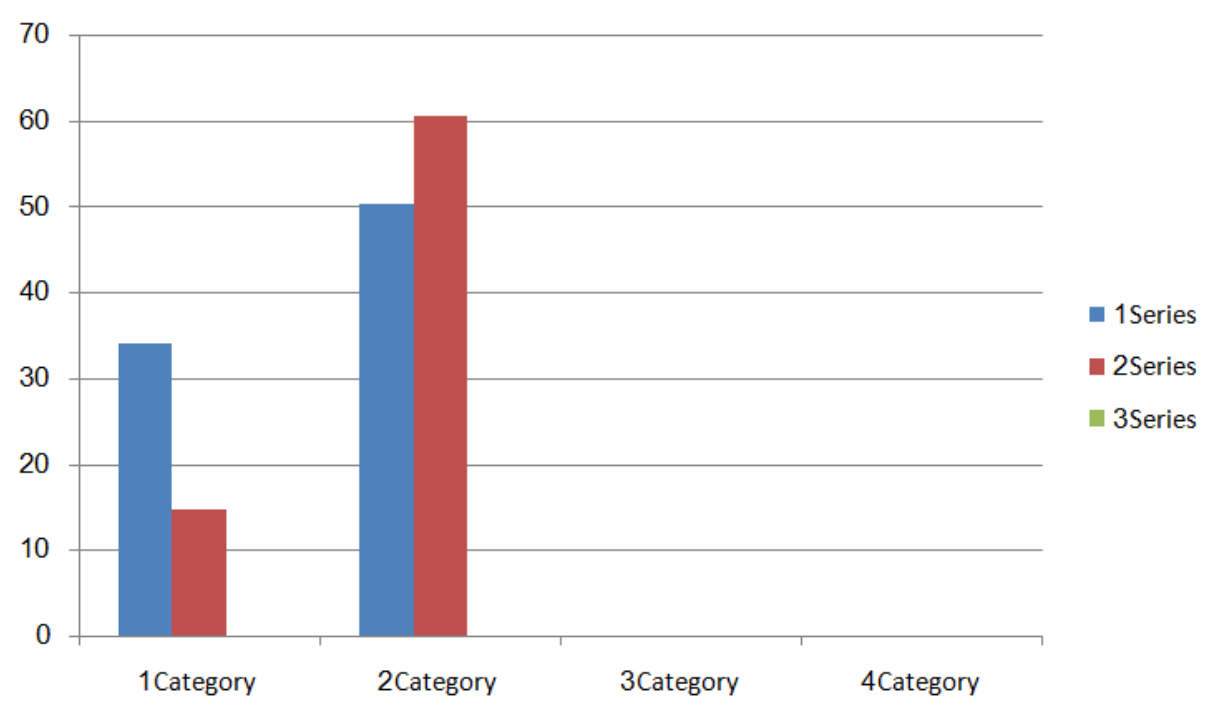

Figure 1: The changes in the rate of vitamin D deficiency \& insufficiency, between academic year 2014-2015 \& 2015-2016 in Gohardasht karaj of Iran.

Category 1: serum vitamin D level < 10; Category 2: serum vitamin D level < 30; Series 1: Academic year 2014-2015; Series 2: Academic year 2015-2016.

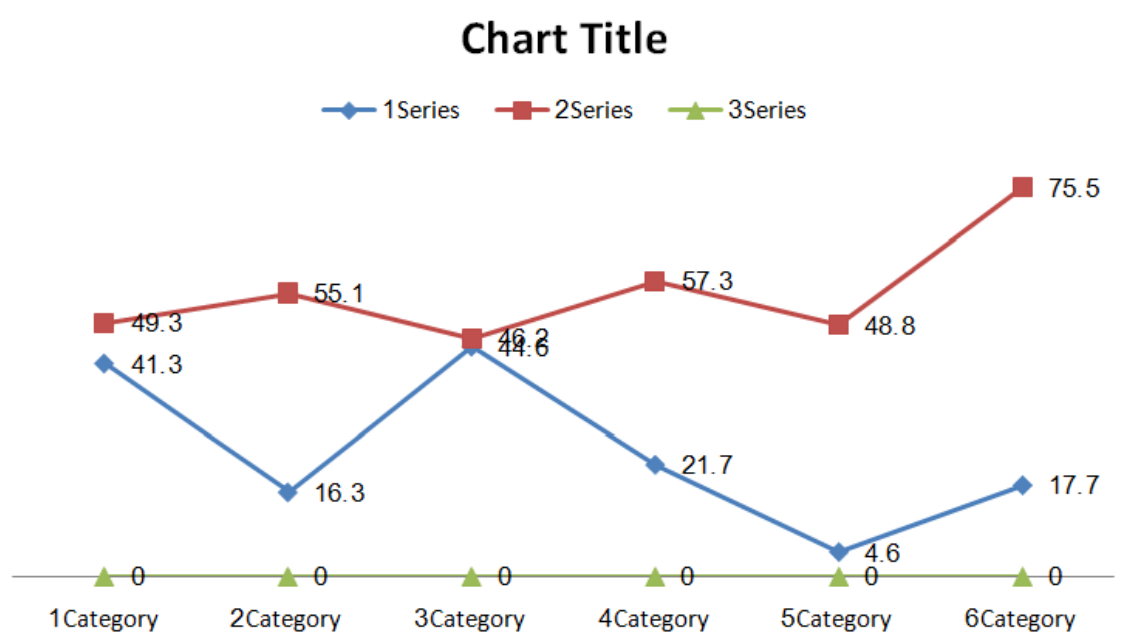

Figure 2: Changes in the prevalence of vitamin D deficiency and insufficiency over time with administration 5000 IU/month vitamin $\mathrm{D}$ to every students.

Series 1: serum vitamin D level < 10; Series 2: 10 <serum vitamin D level < 30; Category 1: OCT 2014; Category 2: DEC 2014; Category 3: FEB 2015; Category 4: OCT 2015; Category 5: DEC 2015; Category 6: FEB 2016. 
Table 1: The rate of medical diseases in the participants, chosen by the system for lab exam, boy's high school in Gohardasht Karaj (above average neighborhood), october 2014.

\begin{tabular}{|c|c|c|c|c|c|}
\hline Participants & Vit $\mathrm{D}<10$ & $10<$ vit $\mathrm{D}<30$ & Vitb12<200 & $200<$ vitb $12<350$ & Anemia \\
\hline \multirow[t]{2}{*}{75} & 31 & 37 & 20 & 43 & 17 \\
\hline & $\% 41.3$ & $\% 49.3$ & $\% 26$ & $\% 57.3$ & $\% 22.6$ \\
\hline participants & $\begin{array}{l}\text { Impaired fasting } \\
\text { glucose level }\end{array}$ & Hypoglycemia & Diabetes & Hypothyroidism & healthy \\
\hline \multirow[t]{2}{*}{75} & 1 & 6 & 0 & 9 & 2 \\
\hline & $\% 1.3$ & $\% 8$ & $\% 0$ & $\% 12$ & $\% 2.6$ \\
\hline
\end{tabular}

Table 2: The rate of medical diseases in the participants, chosen by the system for lab exam, girl's high school in Gohardasht Karaj (above average neighborhood), december 2014.

\begin{tabular}{|c|c|c|c|c|c|}
\hline Participants & vitD $<10$ & $10<$ vitD<30 & Vitb12<200 & $200<$ vitb $12<350$ & Anemia \\
\hline \multirow[t]{2}{*}{49} & 8 & 27 & 1 & 10 & 8 \\
\hline & $\% 16.32$ & $\% 55.1$ & $\% 2.04$ & $\% 20.40$ & $\% 16.32$ \\
\hline participants & $\begin{array}{l}\text { Impaired fasting } \\
\text { glucose level }\end{array}$ & Hypoglycemia & Diabetes & Hypothyroidism & healthy \\
\hline \multirow[t]{2}{*}{49} & 6 & 1 & 2 & 0 & 7 \\
\hline & $\% 12.24$ & $\% 2.04$ & $\% 4.8$ & $\% 0$ & \%14.58 \\
\hline
\end{tabular}

Table 3: The rate of medical diseases in the participants, chosen by the system for lab exam, boy's high school in Gohardasht Karaj (above average neighborhood), February 2015.

\begin{tabular}{|c|c|c|c|c|c|}
\hline Participants & vitD $<10$ & $10<$ vitD $<30$ & Vitb12<200 & $200<$ vitb $12<350$ & Anemia \\
\hline 56 & 25 & 26 & 1 & 6 & 3 \\
\hline$\% 100$ & $\% 44.64$ & $\% 46.42$ & $\% 1.78$ & $\% 10.7$ & $\% 5.35$ \\
\hline participants & $\begin{array}{l}\text { Impaired fasting } \\
\text { glucose level }\end{array}$ & Hypo glycaemia & Diabetes & Hypo thyroids & healthy \\
\hline 56 & 8 & 0 & 0 & 2 & 2 \\
\hline$\% 100$ & $\% 14.28$ & $\% 0$ & $\% 0$ & $\% 3.57$ & $\% 3.57$ \\
\hline
\end{tabular}

Table 4: The rate of medical diseases in the participants, chosen by the system for lab exam, girl's high school in Gohardasht Karaj (above average neighborhood), Oct 2015.

\begin{tabular}{|c|c|c|c|c|c|}
\hline Participants & vitD $<10$ & $10<$ vitD $<30$ & Vitb12<200 & $200<$ vitb $12<350$ & Anemia \\
\hline 115 & 25 & 66 & 9 & 57 & 12 \\
\hline$\% 100$ & $21.7 \%$ & $57.3 \%$ & $7.8 \%$ & $49.6 \%$ & $10.4 \%$ \\
\hline participants & $\begin{array}{l}\text { Impaired fasting } \\
\text { glucose level }\end{array}$ & Hypo glycaemia & Diabetes & Hypo Thyroids & healthy \\
\hline 115 & 2 & 2 & 1 & 3 & 9 \\
\hline$\% 100$ & $\% 1.73$ & $\% 1.73$ & $\% 0.86$ & $\% 2.6$ & $7.8 \%$ \\
\hline
\end{tabular}

Table 5: The rate of medical diseases in the participants, chosen by the system for lab exam, boy's high school in Gohardasht Karaj (above average neighborhood), December 2015.

\begin{tabular}{|c|c|c|c|c|c|}
\hline Participants & vitD $<10$ & $10<$ vitD<30 & Vitb12<200 & $200<$ vitb12<350 & 24 \\
\hline 43 & 2 & 21 & 11 & $\% 55.81$ & 4 \\
\hline$\% 100$ & $\% 4.65$ & $\% 48.83$ & Diabetes & Hypothyroid & healthy \\
\hline participants & $\begin{array}{c}\text { Impaired fasting } \\
\text { glucose level }\end{array}$ & Hypoglycemia & 0 & 9 & 0 \\
\hline 43 & 3 & 0 & 0 & $\% 20.93$ & 0 \\
\hline
\end{tabular}


Table 6: The rate of medical diseases in the participants, chosen by the system for lab exam, girl's high school in Gohardasht Karaj (above average neighborhood), Feb 2016.

\begin{tabular}{|c|c|c|c|c|c|}
\hline Participants & vitD $<10$ & $10<$ vitD $<30$ & Vitb12<200 & $200<$ vitb $12<350$ & Anemia \\
\hline 45 & 8 & 34 & 11 & 24 & 7 \\
\hline$\% 100$ & \%17.77 & $\% 75.55$ & $\% 24.44$ & \%53.33 & $\% 15.55$ \\
\hline participants & $\begin{array}{l}\text { Impaired fasting } \\
\text { glucose level }\end{array}$ & Hypo Glycaemia & Diabetes & Hypo Thyroids & healthy \\
\hline 45 & 0 & 0 & 0 & 3 & 3 \\
\hline$\% 100$ & 0 & 0 & 0 & $\% 6.6$ & $\% 6.6$ \\
\hline
\end{tabular}

Table 7: The mean of vitamin D deficiency \& insufficiency in highschools on Gohardasht-Karaj of Iran during prescription of vitamin D 5000IU/month.

\begin{tabular}{|c|c|c|}
\hline $\begin{array}{c}\mathbf{1 0}<\text { vitamin D<30 } \\
\text { Vitamin D insufficiency }\end{array}$ & $\begin{array}{c}\text { Vitamin D< 10 } \\
\text { Severe vitamin D deficiency }\end{array}$ \\
\hline$\% 46.4$ & $\% 44.6$ & Oct 2014 \\
\hline$\% 55.1$ & $\% 16.3$ & Dec 2014 \\
\hline$\% 49.3$ & $\% 41.3$ & Feb 2015 \\
\hline$\% 57.3$ & $\% 21.7$ & Oct 2015 \\
\hline$\% 48.8$ & $\% 4.65$ & Dec 2015 \\
\hline$\% 75.5$ & $\% 17.77$ & Feb 2016 \\
\hline
\end{tabular}

\section{Discussion}

During two year prescription of 5000 IU vitamin D3/ monthly in high school, we face on 2.5 time decreasing of vitamin D deficiency and a few increasing of vitamin D insufficiency and no case of vitamin D toxicity. By this results we can predict definitely decreasing in vitamin D deficiency \& insufficiency, during a five year program.

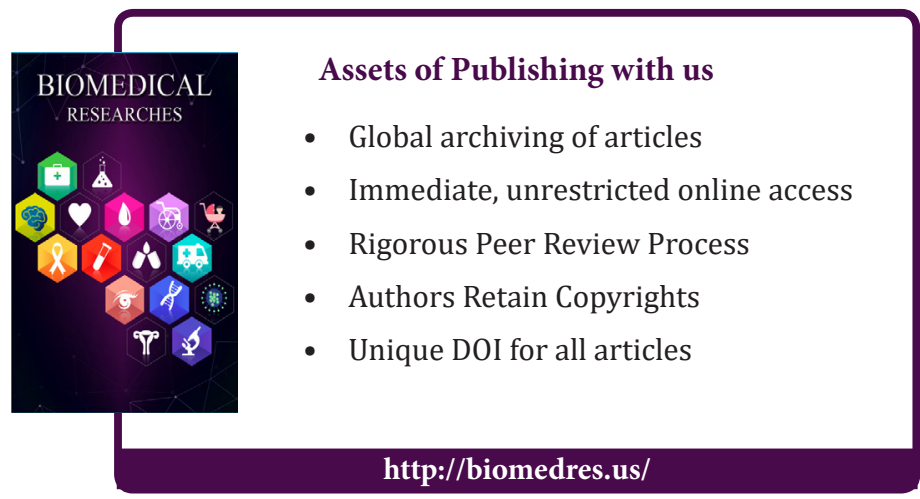

